\title{
LAS CERTEZAS EN TORNO A LA UNIÓN SUDAMERICANA ${ }^{1}$ THE TRUES ABOUT THE SOUTH AMERICAN UNION
}

\author{
Roberto Miranda*
}

RESUMEN

Sudamérica ha mostrado signos de una mayor presencia internacional con respecto a otras épocas. Uno de los aspectos que sostuvo esta presencia ha sido la apelación permanente al concepto de integración. De hecho hubo importantes decisiones políticas y acciones diplomáticas tendientes a estructurar un esquema integracionista. Sin duda que esto creó expectativas. Pero también es cierto que distintas cuestiones han desacelerado y desaceleran la profundización de aquel esquema, a pesar de la retórica $y$ de los gestos que plantean tal intención. Algunas de estas cuestiones condicionan fuertemente lo que es posible y oportuno: la construcción de un poder subregional. Los resultados y conclusiones del estudio de esta situación es lo que desarrollamos en el presente trabajo.

PALABRAS CLAVE: AMÉRICA LATINA * POLÍTICA SUDAMERICANA * INTEGRACIÓN

\section{ABSTRACT}

Recently, South America has shown more international presence than in past times. One feature of this phenomenon has been a continual claim related to the concept of integration. In fact, important political decisions and diplomatic actions tending to the building of an integrationist system have being carried out. Clearly, this has raised expectations. However and despite the positive political signs and rhetorical claims, it is also undeniable that different questions have lost momentum and, consequently, have lessened the velocity of the integrationist project. Some of this questions heavily constraint what is possible and opportune: the construction of a subregional power. In the present work we expose the results and conclusions elicited by the analysis of the current situation.

KEY WORDS: LATIN AMERICA * SOUTH AMERICAN POLICY * INTEGRATION

Consejo Nacional de Investigaciones Científicas y Técnicas, Argentina.miranda@irice.gov.ar

1

Este trabajo condensa algunos resultados y conclusiones del proyecto de investigación que

estamos desarrollando a través del Consejo Nacional de Investigaciones Científicas y Técnicas de Argentina, sobre el papel de este país en la política sudamericana. 


\section{INTRODUCCIÓN}

Sudamérica, en los últimos tiempos, ha registrado realizaciones importantes en cuestiones comunes vinculadas a los derechos humanos, estabilidad democrática, cooperación militar e infraestructura física, por citar algunas. Las coincidencias en torno a estas cuestiones han tenido como telón de fondo la idea de la integración subregional. Por momentos, en los sectores dirigentes esta idea despertó un entusiasmo generalizado en el que se combinaron propuestas $y$ expectativas que imaginaban el nacimiento de un bloque sudamericano, significativo frente a los desafíos del conflicto Norte-Sur.

Pero la expansión de la idea de la integración todavía no eliminó ni redujo la diferencia que existe entre lo que es el compartir cuestiones y lo que es el surgimiento de un actor colectivo subregional. En este trabajo analizamos algunos aspectos de esta diferencia. Partimos de la base de que la identificación y el tratamiento de estos aspectos que acusa la integración sudamericana, es una pista conducente para tener una visión más acabada y efectiva de lo que progresó el subcontinente en torno a esa integración y, sobre todo, de los obstáculos que no sólo han debilitado tal proceso, sino también que han retardado la construcción de un poder subregional.

\section{DE AMÉRICA LATINA A AMÉRICA DEL SUR}

En América Latina, la idea de integración se remonta al siglo XIX cuando hubo diferentes intentos de unión entre los países recién emancipados o en vías de institucionalización estatal. Estos intentos podrían ser explicados desde diversos enfoques disciplinarios y desde diferentes supuestos teóricos. A través de las Relaciones Internacionales es posible señalar que la condición periférica de los países de la región ha sido un factor muy gravitante a la hora en que estos tuvieron que defender un proyecto unionista. Por ejemplo, los fracasos integracionistas que experimentó América Latina durante gran parte de la Guerra Fría pusieron de manifiesto esta realidad, no sólo por influencia de las políticas hemisféricas y de actores extra-continentales, sino también por los desencuentros domésticos que en distintas circunstancias se dieron en la mayor parte de los países de la región.

En la evolución integracionista podemos distinguir, por un lado, los procesos latinoamericanos, y por el otro, los sudamericanos. Esta distinción, en cierto modo, representa la separación que en los últimos tiempos ha estado experimentando América Latina entre lo que es Centroamérica, Caribe y México, y lo que es Sudamérica ${ }^{2}$. La discusión en torno a esta división recién comienza y escapa al propósito de este trabajo, pero es innegable que varias situaciones vinculadas a la política hemisférica $y$ a las relaciones intrarregionales han dado testimonio de esa división.

De todos modos, vale afirmar que los procesos de cooperación sudamericanos se dieron dentro de la lógica integracionista de América Latina, ya que esta región fue el antecedente $y$ el marco sobre el cual aquellos procesos han prosperado, al menos formalmente ${ }^{3}$. Por ello, en primer lugar, sin querer agotar el tema de manera cuantitativa y cualitativa, hacemos un breve recorrido de los intentos latinoamericanos

2 Alguna literatura específica, con cierta arbitrariedad metodológica, emplea la denominación América Latina del norte para incluir a Centroamérica, Caribe y México y, paralelamente, llama América Latina del sur para referirse a Sudamérica. Para Francisco Rojas Aravena (2006:114-130), por ejemplo, existe una clara división de América Latina en distintos sentidos. Por un lado, tenemos una América Latina del norte próxima a Estados Unidos, y por el otro, una América Latina del sur con rasgos autónomos con respecto a Washington. Otro tipo de división, de naturaleza económica y comercial, es entre los países del Pacífico y los del Atlántico. Rojas Aravena también destaca la competencia entre Brasil y Venezuela por influir en la región, como un tercer criterio diferenciador que no excluye los anteriores.

3 Si bien entre los conceptos de cooperación y de integración existen diferencias teóricas, en este trabajo los utilizaremos de manera indistinta basándonos en la naturaleza de sus definiciones que apuntan, en líneas generales, a la convergencia de necesidades comunes y de objetivos compartidos. 
de integración, $y$ luego, trataremos la impronta sudamericana sobre esta cuestión.

\section{A) LOS INTENTOS LATINOAMERICANOS}

En la lógica integracionista de la región podemos considerar dos tipos de procesos, por un lado, los relacionados con la dimensión económica y comercial, $y$ por el otro, los vinculados a la dimensión política. Con respecto a los primeros, que han sido los más por la primacía del carácter militar e ideológico del conflicto Este-Oeste, es muy claro que surgieron como consecuencia de preocupaciones propias de sociedades en desarrollo, y que al mismo tiempo desaparecieron o se empantanaron por múltiples problemas propios de Estados débiles e institucionalmente inestables; en ambos casos, por el origen o por su dinámica, estamos hablando de los países latinoamericanos ${ }^{4}$.

En el caso de la Asociación Latinoamericana de Libre Comercio (ALALC) creada en 1960 a instancias de Argentina, Brasil y México, con el propósito de generar una integración económica de la que siempre se estuvo muy lejos de concretar, con el agravante de que los cuestionamientos estuvieron dirigidos a los aspectos institucionales, sin querer percibir que los inconvenientes eran básicamente políticos. Justamente, las situaciones internas en Argentina y Brasil, por ejemplo, no ayudaron para determinar estrategias orientadas a aquel propósito original.

De la mano del ánimo integracionista se impulsó el surgimiento del Mercado Común Centroamericano (MCC), mediante el Tratado de Managua de 1960 e integrado por Costa Rica, El Salvador, Guatemala, Honduras y Nicaragua, con el fin de relacionar las economías nacionales y apoyar el desarrollo entre los países miembros. También, este ánimo por la cooperación interestatal dio lugar a la firma del Acuerdo

4 Justamente, la característica de sociedad en desarrollo está formulada en clave socioeconómica, mientras que la de Estado débil se plantea desde el punto de vista político. En este caso, vale tener en cuenta las consideraciones de Stephen Krasner (1989). de Cartagena a través del cual nació el Pacto Andino, en 1969, luego llamado Comunidad Andina de Naciones $(\mathrm{CAN})^{5}$. Su desarrollo institucional, que pasó por distintas etapas, en el plano económico fue bastante ambicioso.

Pero lo que obtuvo este bloque estuvo más cerca de las buenas intenciones que de las acciones efectivas. En 1976, el bloque sufrió la defección de Chile durante el gobierno militar de Augusto Pinochet por supuestas incompatibilidades macroeconómicas. En los noventa, la CAN estableció una Zona de Libre Comercio sin la participación de Perú que se había desvinculado transitoriamente, como así también determinó un Arancel Externo Común. Con esto procuró un mayor dinamismo que definitivamente no pudo alcanzar. Luego, contemporáneamente, comenzó una crisis de bloque cuando Colombia, Ecuador y Perú decidieron, cada uno, negociar un Tratado de Libre Comercio (TLC) con los Estados Unidos. Esto produjo la disconformidad de Venezuela que denunció el Acuerdo de Cartagena en abril de 2006 para retirarse de la organización.

También, en 1969, Argentina, Bolivia, Brasil, Paraguay y Uruguay, acordaron el Tratado de la Cuenca del Plata destinado a consolidar el desarrollo y la integración física de este espacio del Cono Sur. Pero este intento sólo fue una expresión diplomática frente a la rivalidad que existía entre Argentina y Brasil traducida en una peligrosa competencia política, militar y nuclear, la cual —obviamente- hacía más que difícil la cooperación subregional, incluso en ese momento ni siquiera se consideró a un nivel intergubernamental la posibilidad de potenciar a la Cuenca del Plata en términos geoeconómicos.

Sin embargo, más allá de esta realidad que con el tiempo se fue convirtiendo en dominante, había otra realidad que con la promesa de la alternativa de la cooperación Sur-Sur como telón de fondo, sostenía para América Latina fundadas expectativas integracionistas. Así apareció la Comunidad del Caribe (CARICOM), creada en 1973 a través del Tratado

\footnotetext{
5 Originariamente el Pacto Andino lo integraron Colombia, Bolivia, Chile, Ecuador y Perú.
} 
de Chaguaramas, con la intención de afianzar la integración económica entre los países miembros $y$, paralelamente, fortalecer las diplomacias de estos países dadas sus características tan especiales con relación, tanto al contexto hemisférico como al mundial ${ }^{6}$. También, en 1978, Brasil decidió cerrar un largo proceso de negociaciones para dar a luz el Pacto Amazónico, que si bien alguna literatura académica lo analizó como una iniciativa propia de la cosmovisión geopolítica de la dirigencia brasileña, lo cierto es que ese pacto fue un medio internacional para tratar de proteger y regular los recursos naturales de que dispone la citada cuenca?.

Pero el espíritu de la integración latinoamericana en clave económica trató de rescatarse $y$ de estimularse a través de la constitución, en 1975, del Sistema Económico Latinoamericano y del Caribe (SELA). Este organismo regional intergubernamental fue propuesto para promover un sistema de consulta $y$ de coordinación de la integración económica, la optimización de la cooperación intrarregional y la definición de estrategias de vinculación con otras regiones del planeta. Ese sistema de consulta $y$ de coordinación no estaba orientado a privilegiar cuestiones comerciales sino cuestiones estructurales de la economía de los países de la región. Para ello, desde el organismo se procuró el diagnóstico de dificultades y la propuesta de alternativas, que finalmente no tuvieron viabilidad por razones cuyo tratamiento ameritaría un trabajo específico.

La insatisfacción generada por la ALALC, principalmente porque no pudo configurar una zona de libre comercio como se había propuesto y porque entró en una

La Comunidad del Caribe, desde 1958, tenía como antecedente a la Federación de las Indias Occidentales Británicas. La Comunidad del Caribe está integrada por Antigua y Barbuda; Barbados; Belice; Dominica; Granada; Guyana; Haití; Jamaica; Montserrat; Sant Kitts and Nervis; Santa Lucía; San Vicente y Granadinas; Surinam; $y$ Trinidad $y$ Tobago.

7 El Pacto Amazónico lo firmaron Brasil, Bolivia, Colombia, Ecuador, Guyana, Perú, Surinam y Venezuela. suerte de parálisis institucional, derivó en la creación de la Asociación Latinoamericana de Integración (ALADI), en 1980. Uno de los objetivos fundamentales de esta organización fue determinar un área de preferencias económicas para conformar un mercado común latinoamericano. En este sentido, la ALADI se formuló con un criterio abierto para permitir la definición de acuerdos de alcance regional y de alcance parcial, la cooperación horizontal con otras estructuras similares en el mundo y una relación específica con experiencias subregionales como la CAN. Sin embargo su performance distó mucho de ser lo que se esperaba de ella. Más aún, ha sido una institución híbrida en el contexto del comercio intrarregional ${ }^{8}$.

Junto a las pretensiones económicas y comerciales de estos procesos de integración, hubo intentos que desde el plano político trataron de responder a la lógica integracionista. En este caso podemos citar la formación del Grupo de Río surgido en un momento muy particular de la llamada Segunda o Nueva Guerra Fría y de la política hemisférica. En efecto, a partir del Grupo de Contadora creado en 1983 que hiciera un aporte significativo para asegurar la paz en Centroamérica, acompañado por el Grupo de Apoyo surgido dos años después, se organizó el Grupo de Río como Mecanismo Permanente de Consulta y Concertación Política ${ }^{9}$. Esta estructura, que comenzó como Grupo de los Ocho en 1986 y en 1990 adquirió la actual denominación, trató diferentes cuestiones de la agenda

8 Para José Miguel Insulza (2003), tanto la ALADI, como la ALALC, fueron instancias de integración complementaria, no competitiva y cerrada, cuando la realidad imponía "coordinar políticas económicas, coordinar esfuerzos y ampliar el espacio económico regional". Por ello, a los organismos latinoamericanos de integración los denominó "retóricos", sin la institucionalidad necesaria para afrontar los desafíos de la política y economía mundiales.

9 El Grupo de Contadora estaba integrado por Colombia, México, Panamá y Venezuela. Por su parte, el Grupo de Apoyo a Contadora lo conformaron Argentina, Brasil, Perú y Uruguay. En los noventa se agregaron Chile, Ecuador, Bolivia, Costa Rica, El Salvador, Guatemala, Honduras, Paraguay, Nicaragua y República Dominicana. 
regional en busca de consensos para la toma de posiciones comunes ante el mundo. Sin embargo, el recorrido del Grupo de Río en cuanto a cuestiones relacionadas con un aspecto clave como es la coordinación política latinoamericana, no ha repondido a las expectativas que se tenían alrededor del papel diplomático que este grupo debía desempeñar ${ }^{10}$.

\section{B) LA IMPRONTA SUDAMERICANA}

Para la perspectiva integracionista de América del Sur debemos tener en cuenta cuatro aspectos. En primer lugar, los antecedentes que hubo sobre la idea de coordinar acciones diplomáticas en pos de objetivos políticos y económicos de carácter subregional. Así podemos remontarnos al proyecto del denominado ABC, en 1915, encarado por Argentina, Brasil y Chile con el propósito de sostener un vínculo de consulta, intercambio de información y resolución de controversias, que finalmente fue desactivado. También, como representativos de la perspectiva integracionista, podemos mencionar las tentativas de Argentina, Brasil y Chile, primero a principios de los cincuenta a través de los presidentes Juan Perón, Getulio Vargas y Carlos Ibáñez, y luego a principios de los sesenta mediante los presidentes Arturo Frondizi, Janio Quadros y Jorge Alessandri, respectivamente.

En segundo lugar, es muy importante considerar el deliberado acercamiento que durante los ochenta se produjo entre los países sudamericanos superando épocas de sospechas diplomáticas mutuas y de tensiones políticas a menudo ligadas a controversias limítrofes. Por ejemplo, la Declaración de Iguazú de 1985 firmada entre los presidentes Raúl Alfonsín y José

10 Paz Verónica Milet (2004), sin embargo, sostiene que "la evaluación de la gestión del Grupo de Río es positiva" como fue su participación en el caso peruano "durante el supuesto fraude electoral cometido por Fujimori". No obstante acepta que el G-Río debe reformular su agenda porque "hay preocupaciones que no deben abandonarse, como son la defensa de la democracia y el debate de respuestas regionales concertadas frente a los nuevos desafíos a la seguridad".
Sarney, fue clave para el fuerte bilateralismo que Argentina y Brasil comenzaron a enhebrar, transformándose en una "alianza estratégica" en 1997 y sosteniéndose ininterrumpidamente a través de los distintos gobiernos democráticos que se fueron sucediendo en ambos países ${ }^{11}$. La proximidad diplomática y la exaltación del concepto de paz en Sudamérica, salvo excepciones como la breve guerra de 1995 entre Perú y Ecuador, sepultó la tradición conflictiva que por mucho tiempo dividió al subcontinente, y abrió amplios espacios de cooperación y negociación mediante una densa vinculación de diferentes cuestiones $^{12}$.

En tercer lugar, el surgimiento del Mercado Común del Sur (Mercosur) mediante el Tratado de Asunción de 1991. Este bloque integrado inicialmente por Argentina, Brasil, Paraguay y Uruguay, fue una clara expresión de las expectativas que en los noventa provocó el concepto de mercado. Por ello, como modelo de integración fue diferente a lo que hasta ese momento se proponía, ya que el Estado dejaba de ser el actor central y promotor de la dinámica integracionista y sólo se limitaba a establecer el marco institucional para que factores transnacionales decidieran el tipo de negocios a llevar a cabo ${ }^{13}$. En esta dirección, el Mercosur

11 Helio Jaguaribe (2005), desde siempre, aseguró categóricamente que el bilateralismo argentinobrasileño era más que fundamental para, en primer lugar, consolidar un poder del Cono Sur, y luego, para estructurar una integración sudamericana. Por ello, para Brasil, la alianza con la Argentina es un objetivo permanente de su política exterior. A pesar de esta convicción, no se debe dejar de lado que discípulos de Jaguaribe pertenecientes al Instituto Brasileiro de Relações Internacionais de Brasilia, sostienen que el poder conosureano y la integración sudamericana dependen del triángulo entre Argentina, Brasil y Venezuela.

$12 \quad \mathrm{Al}$ respecto puede verse el documento que Autores Varios (2004) elaboraron en torno a la combinación de los temas de gobernabilidad, seguridad y diplomacia de las cumbres.

13 Existe un enfoque europeo que señala que el regionalismo latinoamericano de los noventa fue similar al de los sesenta ya que ha sido "impulsado $y$ conducido por iniciativas políticas", $y$ que no han cumplido con "las expectativas que los motivaron, 
trazó un arancel externo común y una zona libre de aranceles con algunas excepciones tratando de caminar hacia una unión aduanera. Por cierto que esta unión, por varias cuestiones, ha sido un objetivo muy lejano ${ }^{14}$. En cambio, en los últimos tiempos, las estructuras estatales comenzaron a tener una mayor participación en la política de integración del Mercosur y esto se ha observado en el reconocimiento que el mundo hizo de esta política al identificar al bloque como un actor subregional de trascendencia internacional.

En cuarto lugar, como consecuencia de la Iniciativa para las Américas de 1990 del presidente George H. W. Bush y del alineamiento político de la Argentina con Estados Unidos impulsado por el gobierno de Carlos Menem, Brasil preparó en 1993 una respuesta subregional que fue la creación de un Área de Libre Comercio de Sudamérica (ALCSA), la cual finalmente no prosperó a pesar de que tres años después volvió a insistir con este proyecto a través del G-Río. Sin embargo, el trabajo diplomático tuvo su fruto. Si bien el ALCSA no se plasmó, Brasil logró reunir a los miembros de la CAN, del Mercosur, más Chile, Surinam y Guyana, en una inédita Cumbre de América del Sur realizada en Brasilia en setiembre de $2000^{15}$.

en particular las relativas a la creación de riqueza y a la disminución de las desigualdades sociales de los países implicados"(Ibáñez, 2000). Justamente, los factores transnacionales estaban comprometidos con la creación de riqueza y no lo cumplieron, mientras los Estados estuvieron ausentes en la formulación de políticas públicas para atenuar los problemas sociales.

Sobre las bases del Mercosur y las dudas que presenta este bloque, entre otros, puede verse: Nicola Phillips (2003).

De esta Cumbre surgió la Iniciativa de Integración Regional Sudamericana (IIRSA) como estructura interestatal de diálogo para promover ejes de integración física e infraestructura de energía, telecomunicaciones y transporte. Esta Iniciativa está integrada por Argentina, Bolivia, Brasil, Chile, Colombia, Ecuador, Guyana, Paraguay, Perú, Surinam, Uruguay y Venezuela.
El éxito de esta iniciativa brasileña se coronó con la creación de la Comunidad Sudamericana de Naciones (CSN) que suscribieron todos los países del subcontinente en Cuzco (Perú), en diciembre de 2004. Un aspecto fundamental de la CSN que merece ser subrayado, fue el haber conciliado las distintas dimensiones que hacen a la integración como la política, económica y no estatal. En esta dirección, los ejes que se impulsaron fueron la concertación y coordinación diplomática, una zona de libre comercio, la integración física, energética y comunicacional, $y$ el relacionamiento intenso con empresas y sociedad civil.

Sin duda que el mayor cuestionamiento que sufrió la formación de la CSN fue el no haber definido una estructura institucional para que le diera gestión política a los ejes de la integración subregional ${ }^{16}$. Principalmente, para que la CSN no quedara registrada en la historia como una mera expresión de voluntades presidenciales propia de una coyuntura, $y$ sí en cambio como una organización interestatal que trascendiera en el tiempo a políticos $y$ gobiernos a través de objetivos y acciones comunes. Es sabido que la diplomacia presidencial sin diplomacia institucional a menudo genera dudas e incertidumbre, aspectos que justamente sobrevuelan el futuro de la $\mathrm{CSN}^{17}$. Un ejemplo de esto tiene que ver con los niveles de aceptación que se mueven en torno al liderazgo brasileño, que si bien pretende ser positivo y constructivo, provoca cierta incomodidad a algunos países y gobernantes del espacio sudamericano ${ }^{18}$.

Ver: Diego Cardona (2005).

17

Las dudas e incertidumbre que sobrevuelan sobre el porvenir de la CSN, referidas principalmente al vacío institucional de este tipo de organización que frena la posibilidad de una estrategia conjunta, es la misma situación que sobrevuela sobre todos los bloques y emprendimientos integracionistas de América Latina (Stuhldreher, 2004).

18 No sólo provoca incomodidad en esferas gubernamentales de algunos países como la Argentina, también genera cierto nivel de molestia y preocupación en sectores empresariales de Venezuela (Giacalone, 2006). 
No obstante vale señalar la relevancia que la CSN ha adquirido para América del Sur, principalmente porque a través de ella la subregión comenzó a buscar una integración e identidad más fuerte, como así también una mayor presencia internacional. El inicio de obras de infraestructura como la Carretera Interoceánica entre Brasil y Perú, o bien el Anillo Energético entre Argentina, Brasil, Chile, Paraguay, Perú y Uruguay, son algunos de los casos concretos que sellan los inicios de la marcha integracionista emprendida bajo la cobertura de la CSN.

\section{3. ¿DE QUÉ INTEGRACIÓN HABLAMOS?}

El largo proceso de convergencia diplomática que han llevado a cabo los países sudamericanos para ir configurando un contexto de integración, respondió a diferentes situaciones. Por un lado, a situaciones referidas al ámbito mundial, y por el otro, a situaciones propias de la subregión.

Con respecto a las primeras, es posible mencionar tanto el escenario internacional que creó la posguerra fría, como la irrupción y expansión que tuvo la globalización ${ }^{19}$. La posguerra fría - iniciada a principios de los noventa-generó un clima favorable a la cooperación e integración intraperiférica, gracias a que se descomprimió la tensión militar e ideológica que mantenían a los países sudamericanos cautivos del conflicto Este-Oeste. De esta forma, estos países pudieron relacionarse intensamente a través de diferentes áreas temáticas como el comercio, recursos naturales, energía, militar, por citar algunos de los más significativos. El clima favorable de la posguerra fría hizo que estos circuitos de cooperación sectorial no fueran vistos como una amenaza a la seguridad continental o a los intereses de la potencia hegemónica.

La globalización también disparó una atmósfera propicia para que los países sudamericanos se encontraran, diplomáticamente, a través de algunos temas que les eran comunes

19 Ver, por ejemplo: Kaus Dingwerth y Philipp Pattberg (2006). a sus respectivas necesidades ${ }^{20}$. Más allá de lo mucho que se ha escrito y discutido en torno a la globalización, esta significó para Sudamérica que la mayor parte de sus países se conectaran entre sí a través de las inversiones y negocios que realizaban distintos actores transnacionales. Dejando de lado la polémica que originaron las acciones desarrolladas por estos actores bajo el paraguas de la globalización, es evidente que en América del Sur sus transacciones empujaron a los países hacia distintos niveles de interdependencia que, si bien en muchos casos pusieron de manifiesto amplias asimetrías, dieron lugar a una integración hasta ese momento desconocida.

Por su parte, las situaciones propias del subcontinente tuvieron que ver con un aspecto político como ha sido la redemocratización de los ochenta, y con un aspecto cultural como ha sido el grado de concientización que las sociedades civiles de los países sudamericanos fue logrando en relación con la integración. Sobre la redemocratización podemos afirmar que fue un factor prácticamente decisivo para que Sudamérica iniciara una nueva etapa en las relaciones diplomáticas entre sus países. Este hecho permitió zanjar diferencias que en el pasado contrariaban a algunos Estados bajo determinadas hipótesis de conflicto, como así también posibilitó que varios países conformaran alianzas estratégicas entre sí para obtener fortalezas ante terceros que difícilmente tendrían de manera aislada. Paralelamente, a pesar de la precariedad de algunos regímenes políticos, la democracia se ha transformado en un elemento esencial para la estabilidad de la subregión, no tanto por la percepción que sobre ella se sostuvo desde otras partes del mundo, sino por la necesidad que tuvieron muchos países de esta subregión de reducir o eliminar su vulnerabilidad internacional.

Además de la integración impulsada desde las diferentes esferas gubernamentales a través de las instituciones democráticas, esta integración — con el paso del tiempo- ha estado sostenida mediante una alta valoración que

En torno a la globalización desde una mirada sudamericana, ver: Hugo Fazio (2006). 
las sociedades civiles sudamericanas fueron estructurando sobre la misma. El imaginario colectivo de hostilidad hacia el vecino que en el contacto entre "nosotros" y "los otros" era de rechazo y hasta de anulación, muchas veces utilizado por las élites y los gobiernos para justificar sus mezquindades y prestarse al divisionismo estimulado por factores externos, cedió ante una creciente convicción ciudadana basada en el entendimiento y la cooperación intersocietal. Esta convicción se fue convirtiendo en una cultura pública de las sociedades civiles sudamericanas, testimoniando un sentido de pertenencia subregional absolutamente inédito con respecto a la historia de estas sociedades ${ }^{21}$.

A pesar de estas situaciones favorables a la integración sudamericana, $y$ de lo que hasta ahora ha sido su impronta de acuerdo con lo desarrollado precedentemente, la subregión está lejos de conformar un formato institucional si nos guiamos por los patrones teóricos y por la experiencia europea ${ }^{22}$. Lo que existe en América del Sur es un estilo político tendiente a la integración que surge de una combinación de principismo $y$ de pragmatismo.

Es sabido que la fuente fundamental del principismo integracionista es el proyecto unionista que pretendió motorizarse cuando en el siglo XIX los Estados sudamericanos intentaban confundirse en una nación, o en muy pocas naciones. Obviamente que en este proyecto, la integración era considerada como un postulado esencial e inevitable. En nuestra época, el discurso oficial del estilo político que busca inclinarse hacia la integración se ha apoyado en conceptos unionistas. En cambio, en el nivel de las acciones políticas y diplomáticas, estos conceptos no han tenido la misma prioridad que en el discurso porque ante la naturaleza cambiante de los diferentes contextos de las relaciones internacionales, fue asomando el pragmatismo en las diplomacias sudamericanas. Esta

Este proceso cumple con algunos de los requisitos que Monserrat Guibernau (1996) define como clásicos de lo que es la "política de identidad" de un Estado. realidad se ha sintetizado en una tensión entre lo que representa la ideología de la unión sudamericana $y$ lo que son las relaciones de poder en el espacio subregional, tensión de la cual surgió la integración como eje estructurante y que hasta el momento no pudo más que conformar un regionalismo limitado.

\section{EL POR QUÉ DE UN REGIONALISMO LIMITADO}

El regionalismo de América del Sur, aunque haya sido limitado, creó expectativas de todo tipo en torno a la integración. De hecho hubo cuestiones que fueron resueltas parcial o totalmente, como crisis institucionales, conflictos en áreas de frontera, integración energética, protección de medio ambiente, cooperación militar, por citar algunas ${ }^{23}$. Hubo innumerables muestras de voluntad política para sostener esta tendencia integracionista. Pero también hubo frenos a la idea de la integración sudamericana. Un aspecto relacionado con esta cuestión es el reducido nivel de influencia que la integración ha tenido sobre las políticas exteriores de los países de la subregión.

Las políticas no sólo han sido reticentes a subordinarse a las reglas formales de la integración, sino que la mayor parte de estas han considerado a este proceso como un recurso para el posicionamiento internacional de su país, o bien para tener más poder en una negociación diplomática con terceros. Así podemos señalar - por ejemplo- que el Mercosur fue el único espacio en el cual la Argentina pudo apoyarse durante el default para no profundizar su depreciación como actor internacional. También, podemos afirmar que para Brasil este bloque ha sido fundamental en su relación bilateral con Estados Unidos al oponerse al Área de Libre Comercio de las Américas (ALCA). Otro caso más es Venezuela, que ha logrado trascender internacionalmente gracias a la gravitación que Sudamérica tiene como esquema de integración, porque desde esta perspectiva otras (2003). 
al presidente Hugo Chávez le ha sido más fácil proponer su proyecto político.

De una u otra forma, las políticas sudamericanas han hecho uso del supuesto integracionista para obtener beneficios propios sin considerar seriamente el espíritu unionista. La lógica de varios países ha sido la clásica lógica de los intereses nacionales, cuya consecuencia directa fue quitarle primacía a los intereses subregionales. Esta suerte de mezquindad no sólo puso de manifiesto el escaso impacto que la integración ha tenido en la formulación de muchas de las políticas exteriores; también puso en evidencia que la coordinación y concertación intergubernamentales, en la mayor parte de las áreas temáticas, no ha podido superar la diplomacia declarativa testimoniada a través de diferentes cumbres y reuniones del subcontinente.

La falta de compromiso de las políticas exteriores sudamericanas para sostener una diplomacia efectivamente integracionista, se puede apreciar en la relación de la subregión con Estados Unidos. La condición de variable interviniente que este país porta históricamente en los diversos asuntos sudamericanos es indiscutible ${ }^{24}$. En este caso, lo más significativo no ha sido la incidencia de Washington sobre América del Sur, como sí lo ha sido el carácter indeterminado que tuvo esta incidencia en la región ${ }^{25}$. Justamente, en estos momentos en los que el espacio sudamericano ha tratado de construir pistas integracionistas independientemente de las estrategias de Washington, Estados Unidos aún divide a los países de la subregión por el tipo de relación que cada uno de estos quiere tener con él ${ }^{26}$.

24 Al respecto cabe agregar que esta realidad, en gran medida, determina buena parte del discurso de política exterior de la mayor parte de los países latinoamericanos. La academia norteamericana, en especial, refleja permanentemente esta situación. Ver, por ejemplo, Joseph Tulchin (2004).

Sobre esta cuestión ver, entre otros autores: Peter Hakim (2006).

Gordon Mace y Hugo Loiseau (2005), plantean algunas consideraciones téoricas interesantes en torno al debate sobre el rol de Estados Unidos en la cooperación hemisférica.
Los tratados bilaterales de libre comercio (TLC) han sido un ejemplo del diseño de la política norteamericana para quebrar los intentos de regionalismo, los cuales se inscribían en un esquema de refuerzo de las relaciones Sur$\mathrm{Sur}^{27}$. Por efecto de las tentaciones que despiertan los TLC y de los desaguisados endógenos en la mayor parte de los países sudamericanos por conflictos intraestatales como ha sido el caso de Ecuador, la subregión se ha fraccionado parcialmente sin llegar a sucumbir de manera absoluta como si estuviésemos frente a una desintegración, de acuerdo con lo que han sugerido algunos análisis ${ }^{28}$. Por eso, el dilema de los países subregionales está centrado, por un lado, en que es inevitable el tener que considerar a Estados Unidos dentro de las dimensiones comercial y militar, $y$ por el otro, en que la cooperación Sur-Sur debería llevarse a cabo sin la injerencia de Washington ${ }^{29}$.

El afán por superar este dilema hizo que algunos países sudamericanos se movieran en

Se dice que los intentos periféricos de regionalismo internacional han creado alianzas entre los países en desarrollo que, de un modo u otro, tienden a trastocar el equilibrio de poder del norte. Estas alianzas son las que se han formado en el ámbito de la Organización Mundial del Comercio para sostener posiciones comunes; ver Dot Keet (2006).

28 Abraham Lowenthal (2006:63-77) afirma categóricamente que Estados Unidos divide su relación con América Latina y el Caribe en estrategias bilaterales $y$ subregionales, $y$ que lo hace en términos económico-comerciales dejando en un plano secundario los aspectos ideológicos y militares. De este forma determina tres áreas. Una es México, América Central y el Caribe, otra es el área andina, y por último el Cono Sur. A partir de esta división entre la zona andina y el Cono Sur, la política norteamericana decide qué prioriza y cómo coopta a los distintos países, lo cual -inevitablemente-impacta en los intentos regionalistas sudamericanos.

29 Si bien Riordan Roett (2006:110-125) en su análisis se refiere a la estructura de relaciones entre Estados Unidos y América Latina, cuando destaca la "creciente presencia de China" en la región y la pretensión de Venezuela de participar en la política mundial, como cuestiones "negativas", traduce la preocupación de sectores dirigentes norteamericanos por el futuro de Sudamérica, entendido esto en clave de control subsistémico. 
el extremo de confrontar sistemáticamente con los Estados Unidos, o bien en el extremo opuesto de adscribir a los objetivos norteamericanos, a veces de manera incondicional. Esta realidad no ha sido favorable a la integración subregional, porque de una forma $u$ otra Washington se queda con las "ganancias relativas" ante la ausencia de cohesión de bloque, si es que podemos emplear esta expresión de modo laxo para lo que es la política del subcontinente ${ }^{30}$. En otras palabras, tanto la relación diferente de cada país sudamericano con Estados Unidos, como el vacío de una coordinación política autosostenida y representativa de la subregión, han desacelerado la integración empujándola a un regionalismo limitado.

\section{CUESTIONES DE POLÍTICA SUDAMERICANA}

Se puede afirmar que desde la Cumbre de Presidentes de América del Sur del 2000, la subregión fue configurando una política propia en torno a la idea de integración. Esta política de algún modo le asignó un perfil internacional a Sudamérica, teniendo en cuenta que este espacio siempre ha sido irrelevante para los decisores de la agenda mundial. Como señalamos más arriba, la convergencia unionista en la subregión ha tenido y tiene avances e imperfecciones en relación con lo que debe ser un proceso de integración mayor ${ }^{31}$. No obstante Sudamérica logró cambiar de actitud: de una posición pasiva pasó a una muy activa impulsando acciones externas comunes motorizadas

Si bien Nicola Phillips (2005) se refiere al contexto de la relación de Estados Unidos con América Latina, su trabajo tiene valor en términos conceptuales para analizar más concretamente el futuro de Sudamérica, sobre todo a partir de lo que él denomina como nuevo bilateralismo.

Un proceso de integración mayor se refiere a lo que la teoría de las relaciones internacionales señala como la coronación de un actor colectivo supranacional, luego de que los actores estatales involucrados cumplan con determinadas etapas para su formación, como por ejemplo la de supeditar institucionalmente ciertos intereses nacionales a los objetivos del interés regional. por necesidades, problemas y urgencias, sobre condiciones armónicas y también sobre condiciones conflictivas, en busca de sistemas de cooperación intrarregional.

A nuestro entender, las dificultades para materializar la idea de integración no radican tanto en la ineficacia por resolver cuestiones vinculadas a las estructuras y procedimientos de institucionalización que la literatura específica suele subrayar, como sí en cambio en el tratamiento de algunos aspectos fundamentales ligados a la política sudamericana. En este sentido es posible tener en cuenta tres aspectos.

Primero, el liderazgo regional que Brasil decidió acentuar a partir del protagonismo internacional del presidente Luiz Inácio Lula da Silva. Como afirmamos más arriba, la creación de la CSN ha sido todo un emblema político de este liderazgo que tiene en la diplomacia multilateral a una de las herramientas fundamentales de la que se vale Brasil para irradiar su condición de líder $^{32}$. Una de las muestras entre varias de esta condición, ha sido su participación en las crisis institucionales tanto de Bolivia en octubre de 2003, como de Ecuador en mayo de 2005. Desde un punto de vista, se dice que el liderazgo brasileño es positivo para "ordenar" la interlocución de Sudamérica con el resto del mundo. Desde otra perspectiva - en cambio- se habla del riesgo de que Brasil se transforme en un líder con roles hegemónicos teniendo en cuenta que en la dimensión estratégico-militar es un Estado pivot de Estados Unidos ${ }^{33}$.

Justamente, Washington se ha preocupado por destacar su preferencia por Brasil en el marco de la política sudamericana ${ }^{34}$. Sobre esto

32 Ver: María Soares de Lima y Mónica Hirst (2006). Riordam Roett (2003) no cree que Brasil esté en condiciones de liderar la subregión y convertirse en un jugador global, a pesar de ser un mercado emergente $y$ un actor internacional clave en la política latinoamericana.

33 Este tema lo hemos desarrollado en un trabajo específico sobre las relaciones triangulares entre Argentina, Brasil y Estados Unidos (Miranda, 2004).

$34 \quad$ Un dato que puede ser considerado reciente y muy representativo, fueron las expresiones categóricas 
es posible realizar distintas especulaciones ${ }^{35}$. Pero el énfasis puesto por la diplomacia norteamericana tiene que ver con el objetivo de frenar la expansión del discurso y de las acciones que Chávez impulsa en la mayor parte de los países sudamericanos. Más allá de esta apreciación, es cierto que Venezuela ha competido subyacentemente con Brasil en algunos espacios de poder de la realidad de América del Sur. De algún modo, Venezuela ha tratado de quitarle fuerza al liderazgo brasileño aún en los contextos de cooperación e integración que se dieron en la subregión. Pero la dificultad está en que a pesar de las enormes diferencias entre Brasil $y$ Venezuela en términos de potencia regional, es posible la hipótesis de la lucha por la influencia en Sudamérica aunque esto se restrinja al campo de lo simbólico.

Segundo, la mayor parte de la evolución de la integración sudamericana, que fue fruto de una típica matriz de interdependencia a través de la cooperación sectorial, se movió en torno a asimetrías que por ser estructurales de alguna forma han segmentalizado a la subregión. Las coincidencias y acuerdos que en diversas áreas temáticas tuvieron casi todos los países del subcontinente, no han sido suficientes para eclipsar las necesidades que tienen algunos de estos países que están en busca de su viabilidad, como fue el caso de Bolivia. Su política sobre hidrocarburos sensibilizó significativamente sus relaciones con Argentina $y$ Brasil, por citar un ejemplo. En una medida considerable, las asimetrías estructurales han sido un fuerte obstáculo para la cooperación Sur-Sur en el contexto sudamericano, y lejos de concretarse en este contexto mecanismos compensatorios al estilo de la Unión Europea,

de la secretaría de Estado de Estados Unidos, Condolezza Rice, cuando en abril de 2005 visitó Brasilia y señaló que Brasil estaba en el mismo nivel internacional que China e India y que en el futuro cercano se transformaría en una potencia mundial.

35 Para algunos analistas, como es el caso de Andy Klom (2003:351-368), Brasil, piloteando el Mercosur, está en una encrucijada en su relación, por un lado con Estados Unidos, y por el otro con la Unión Europea. esas asimetrías se convirtieron en una pieza disfuncional para materializar la integración subregional.

Tercero, esta integración se ha caracterizado por mostrar una vocación multilateral mediante la retórica política y diplomática, cuando en realidad se sostuvo y se sostiene mediante una red de bilateralismos fuertes ${ }^{36}$. A título de ejemplo vale citar las "alianzas estratégicas" entre Argentina y Brasil; Brasil y Venezuela; Chile y Argentina; Perú y Brasil; Venezuela y Argentina; Chile y Brasil; y Ecuador y Chile. Esta realidad ha puesto en claro que la expectativa por encolumnar a los países sudamericanos detrás de la estructuración de intereses regionales no prosperó como se deseaba o proclamaba. Esta situación no sólo reforzó la estratificación sudamericana beneficiando a los países internacionalmente mejor posicionados, sino también ha sido motivo para que la diferenciación intrarregional fuera mayor y mostrara claros indicios de debilidad ante actores poderosos.

\section{A MODO DE CIERRE}

Un balance sobre la integración sudamericana ameritaría otro tipo de trabajo. Para esto habría que revisar todas y cada una de las dimensiones de este contexto subregional a través del análisis pormenorizado de las distintas áreas temáticas. En cambio, por lo desarrollado hasta aquí podemos concluir que la idea de integración en América del Sur se ha plasmado en realidades. Diversas acciones relacionadas con la diplomacia, el comercio y la seguridad en las que se involucró la mayor parte de los países de la subregión, han sido claves para los avances que Sudamérica alcanzó tratando de erradicar el desencuentro y la indiferencia que en el pasado fueron moneda corriente.

Sin duda que estos avances no han eliminado las discordias y las fisuras intrarregionales.

Esta hipótesis de trabajo la desarrollamos detalladamente en el III Congreso de Relaciones Internacionales, realizado en el Instituto de Relaciones Internacionales de la Universidad Nacional de La Plata, Argentina (Miranda, 2006). 
Mucho menos la desproporción entre los países del subcontinente, no sólo de índole material, también en relación con los objetivos diplomáticos y con las cuestiones culturales. Por un lado, percibimos el hecho positivo de que los países sudamericanos consideraran su sentido de pertenencia a través de la configuración de una identidad internacional que cada vez más pone a la subregión en los asuntos mundiales, $y$ por el otro, observamos una situación muy negativa como ha sido la de no contar con una coordinación política sistemática para afrontar las agendas externas desde una estrategia común.

Esta falencia hace difícil el manejo de las fragmentaciones en las que suele caer la subregión y que, eventualmente, favorecen el divisionismo y la escisión entre los países. Es uno de los peores síntomas, porque torna a los países sudamericanos en vulnerables y más dependientes de lo que lo son. Pero la consecuencia mayor está en que retarda la construcción de un poder subregional, que es claramente necesario para los países sudamericanos ante los cambios $y$ las tendencias que viene experimentando el mundo.

\section{REFERENCIAS BIBLIOGRÁFICAS}

Autores Varios. Gobernabilidad, seguridad y diplomacia de Cumbres. Santiago: Flacso-Chile; Department of Foreign Affairs and International Trade de Canadá, 2004.

Botto, Mercedes y otras. "El nuevo escenario político regional y su impacto en la integración". Nueva Sociedad 186. 2003: 114-127.

Cardona, Diego. "¿Tiene futuro la comunidad sudamericana de naciones?". Foreign Affairs en Español 5. 2005: 2.

Dingwerth, Kaus y Pattberg, Philipp. "Global Governance a Perspective on World Politics". Global Governance 12. 2006: 185-203.
Fazio, Hugo. "La historia global: ¿encrucijada de la contemporaneidad?". Revista de Estudios Sociales 23. 2006: 59-72.

Fawcett, Louise. "Exploring regional domains: a comparative history of regionalism". International Affaire 80. 2004: 429-446.

Giacalone, Rita. "La Comunidad Sudamericana de Naciones: ¿una alianza entre izquierda y empresarios?". Nueva Sociedad 202. 2006: 74-86.

Guibernau, Monserrat. Los nacionalismos. Barcelona: Ariel, 1996.

Hakim, Peter. "Is Washington Losing Latin American?” Foreign Affaire. 2006: 85/1.

Ibáñez, Joseph. "El nuevo regionalismo latinoamericano en los años noventa". Iberoamérica ante los procesos de integración. Actas de las XVIII Jornadas de la Asociación Española de Profesores de Derecho Internacional y Relaciones Internacionales. Madrid. 2000: 319-328.

Insulza, José. “Es posible una visión renovadora para orientar el futuro de América Latina?". Estudios Internacionales. Santiago. 2003: 140.

Jaguaribe, Helio. "Alianza argentino-brasileña". Diplomacia, estrategia, política 2. Proyecto Raúl Prebish. 2005: 43-53.

Keet, Dot. Alternativas estratégicas Sur-Sur al sistema económico y régimen de poder globales. Amsterdam: Transnational Institute, 2006.

Klom, Andy. "Mercosur and Brazil: a European perspective". International Affairs. 2003: 351-368.

Krasner, Stephen. Conflicto estructural. El tercer mundo contra el liberalismo 
global. Bs. As.: Grupo Editor Latinoamericano, 1989.

Lowenthal, Abraham. "De la hegemonía regional a las relaciones bilaterales complejas: Estados Unidos y América Latina a principios del siglo XXI". Nueva Sociedad 206. 63-77.

Mace, Gordon y Loiseau, Hugo. "Cooperative Hegemony and Summitry in the Americas". Latin American Politics and Society 47.4. 2005: 107-134.

Milet, Paz Verónica. "El Grupo de Río en el nuevo escenario internacional". Diplomacia 61. Academia Diplomática de Chile. 2004: 117-130.

Miranda, Roberto. "Hegemón y pivot: una combinación de presiones en torno a la agenda externa de la Argentina". Políticos 3. Instituto de Ciencia Política, Universidad Católica de Santa Fe. 2004: 77-95.

"Subregionalismo y Argentina: de lo ilusorio a lo posible". III Congreso de Relaciones Internacionales. Instituto de Relaciones Internacionales, Universidad Nacional de La Plata, 2006.

Phillips, Nicola. "The rise and fall of open regionalism? Comparative reflections on regional governance in the Southern Cone of Latin America". Third World Quarterly 24.2. 2003: 217234.
. "U.S. Power and the Politics of Economic Governance in the Americas". Latin American Politics and Society 47.4. 2005: 1-25.

Roett, Riordam. "El papel de Brasil como potencia regional". Varios Autores. América Latina en un entorno global en proceso de cambio. Bs. As.: Nuevohacer, Grupo Editor Latinoamericano. 2003: 227-248.

. "Estados Unidos y América Latina: estado actual de las relaciones". Nueva Sociedad 206. 2006: 110-125.

Rojas Aravena, Francisco. "El nuevo mapa político latinoamericano. Para repensar los factores que marcan las tendencias políticas". Nueva Sociedad 205. 2006: 114-130.

Soares de Lima, Maria y Hirst, Mónica. "Brazil as an intermediate state and regional power: actino, choice and responsibilities". International Affairs 82.1. 2006: 21-40.

Stuhldreher, Amalia. "La regionalización como estrategia frente a la globalización. La concepción de política externa conjunta en los bloques de América Latina y el Caribe". Estudios Internacionales. Santiago, 2004: 145.

Tulchin, Joseph. "América Latina en el sistema internacional". Agenda Internacional 2. Bs.As. 2004: 32-43. 
